Turpin, R., Lejeune, J.: Les chromosomes humains. Paris: Gauthier Villars 1965. Vogel, W.: Identification of $G$ group chromosomes involved in a $G / G$ tandemtranslocation by the Giemsa-band technique. Hum. Genet. 14, 255-256 (1972)

Paris Conference - 1971. Standardization in human cytogenetics - The National Foundation, March of Dimes. vol. VIII No 7 (1972)

Reçu le 16 mai 1973 / Accepté par H. Bauer Bon à imprimer le 19 mai 1973

Prof. J. Lejeune, Dr. M. Prieur Chaire de Génétique Fondamentale Institut de Progénèse 15, rue de l'Ecole de Médecine Paris 75006

France
Dr. B. Dutrillaux

Chargé de Recherches C.N.R.S.

Institut de Progenèse

Dr. M. O. Rethoré

Maitre de Recherches INSERM

Institut de Progénèse

\title{
Druckfehler/Printing Errors
}

\begin{tabular}{|c|c|c|c|c|c|}
\hline Band & Seite & Abschnitt & Zeile & lies & anstatt \\
\hline Volume & page & paragraph & line & read & instead of \\
\hline \multirow[t]{4}{*}{42} & 158 & 2 & 5 & of & fo \\
\hline & 407 & Table & 4 & AA & AA 2 \\
\hline & 411 & 4 & 5 & noticeable & noticeable \\
\hline & 417 & & 24 & 17 & 7 \\
\hline \multirow[t]{14}{*}{43} & 50 & 1 & 2 & Fibrillen & Fribillen \\
\hline & 90 & Table & 3 & with respect & with-respect \\
\hline & 106 & Legend & 4 & $\mathrm{X}_{2}$, the $\mathrm{A}_{2}$ & $X_{2}$, the $A_{1}$ \\
\hline & 112 & 1 & 3 & $l$ & 1 \\
\hline & 153 & 2 & 2 & discrete & descrete \\
\hline & 241 & 3 & 3 & design & designe \\
\hline & 244 & 2 & 3 & model & modell \\
\hline & 247 & Abstract & 15 & therefore & therefore the \\
\hline & 291 & 2 & 3 & the most & most of the \\
\hline & 292 & 3 & 3 & from each & each \\
\hline & 307 & 2 & 3 & total & toal \\
\hline & 319 & 1 & 3 & Woollam & Woolam \\
\hline & 324 & 1 & 3 & others & other \\
\hline & & 2 & 9 & isolated & isolatet \\
\hline
\end{tabular}

